\title{
Robust Adaptive PID Control of Robot Manipulator with Bounded Disturbances
}

\author{
Jian Xu and Lei Qiao \\ Department of Automation, Harbin Engineering University, Harbin 150001, China \\ Correspondence should be addressed to Lei Qiao; qiaolei2008114106@gmail.com
}

Received 6 July 2013; Accepted 1 October 2013

Academic Editor: Zhiguang Feng

Copyright (C) 2013 J. Xu and L. Qiao. This is an open access article distributed under the Creative Commons Attribution License, which permits unrestricted use, distribution, and reproduction in any medium, provided the original work is properly cited.

To solve the strong nonlinearity and coupling problems in robot manipulator control, two novel robust adaptive PID control schemes are proposed in this paper with known or unknown upper bound of the external disturbances. Invoking the two proposed controllers, the unknown bounded external disturbances can be compensated and the global asymptotical stability with respect to the manipulator positions and velocities is able to be guaranteed. As compared with the existing adaptive PD control methods, the designed control laws can enlarge the tolerable external disturbances, enhance the accuracy in finite-time trajectory tracking control, and improve the dynamic performance of the manipulator systems. The stability and convergence properties of the closedloop system are analytically proved using Lyapunov stability theory and Barbalat's lemma. Simulations are performed for a planner manipulator with two rotary degrees of freedom to illustrate the viability and the advantages of the proposed controllers.

\section{Introduction}

Robot manipulators play an important part in modern industry by providing lower production cost, enhanced precision, quality, productivity, and efficiency. The control of rigid robot manipulators faces significant difficulties such as highly nonlinear, coupled, and time-varying behaviors. Moreover, there always exist uncertainties in the system's dynamic model, such as the external disturbances and parameter uncertainty, to name a few, which cause unstable performance of the robot manipulator systems.

Since linear control methods are not suitable for strong coupled, nonlinear, and time-varying rigid robot manipulator systems, many nonlinear control schemes based on conventional PID control theory have been proposed to improve the control performance. In [1], the global asymptotic stability of a class of nonlinear PD-type controllers for position and motion control of robot manipulators is analyzed, and a global regulator constrained to deliver torques within prescribed limits of the actuator's capabilities is proposed. This class of controllers, when rule-based or gain scheduling approaches are used, can get high performance control systems. However, it has been shown that although the PD controller is robust with respect to uncertainties on inertial parameters and the global asymptotic stability is guaranteed, uncertainties on the gravity parameters may lead to undesired steady-state errors [2]. A PID control scheme can eliminate the steady-state errors, but it can only ensure local asymptotic stability. Moreover, to guarantee the stability, the gain matrices must satisfy complicated inequalities [3]. In [4], a new variable structure PID control scheme is designed for robot manipulators. Even through the global asymptotic stability of the controlled robot systems is analyzed, the bounds of system parameter matrices need to be known in the controller design.

To further enhance the tracking performance of robot manipulator systems in presence of parametric uncertainties, significant efforts have been made to seek advanced control strategies. Robust and adaptive control schemes of robot manipulators have been the active research topics for many years. Robust control laws are used for external disturbances, unstructured dynamics, and other sources of uncertainties. Leitmann [5] and Corless and Leitmann [6] present a popular approach utilized for designing robust controller for robot manipulators. In an early application of the Corless-Leitmann approach to robot manipulators [7], a simple robust nonlinear control law is derived for $n$-link robot manipulators using the well-known Lyapunov 
based theory of guaranteed stability of uncertain systems. The uncertainty bounds needed to derive the control law and to prove that uniform ultimate boundedness of the tracking errors only depends on the inertia parameters of the robot. Some other robust control methods developed based on $[5,6]$ are given in $[8,9]$. However, disturbances and unmodeled dynamics are not considered in the algorithms in [7-9]. In [10], Spong's method [7] is extended in such a manner that the control law is robust not only to uncertain inertia parameters but also to unmodeled dynamics and disturbances. Another improvement to the Spong's methodology [7] is suggested in [11]. A drawback of a single robust control is that it cannot estimate the uncertainties and disturbances online, which limits the adaptability of the controller to the changed uncertain parameters. Adaptive algorithm provides an effective way to solve this problem; however, most adaptive controls, like most parameter adaptive methods, may exhibit poor robustness to unstructured dynamics and external disturbances. Some related results can be seen in [12-15]. To resolve this, a combination of robust control and adaptive algorithm is investigated in a number of literature sources. In [16], adaptive controllers are designed for robot manipulator systems that yield robust trajectory in spite of the unwanted effects of the external disturbances and fast maneuvering of the manipulator. The convergence rate is improved and the transient oscillation is reduced considerably. In [17], an adaptive control law for continuous-time direct adaptive control of robot manipulator is presented. The algorithm is suitable for swift adaptation to rapidly changing system parameters. And the uniform global asymptotic stability with respect to the manipulator positions and velocities is guaranteed for unknown constant parameters. In [18], a decentralized adaptive robust controller is investigated for trajectory tracking of robot manipulator systems. A disturbance observer (DOB) is introduced in each local controller to compensate for the low-passed coupled uncertainties, and an adaptive sliding mode control term is employed to handle the fast-changing components of the uncertainties beyond the pass band of the DOB. For some other results on robust and adaptive control the reader can refer to [19-22].

Furthermore, other control algorithms such as fuzzy logic, neural networks, and PD control have been adopted to combine with robust and adaptive control to cope with the problems in robot manipulators control. In [23], a robust adaptive compensation scheme is presented for compensation of asymmetric deadzone, dynamic friction, and uncertainty in the direct-drive robot manipulator. The estimation laws of deadzone and friction are proposed to offset both deadzone of joint input torque and friction. A model-free recurrent wavelet cerebellar model articulation controller (RWCMAC) to mimic the ideal control law is employed to overcome some shortcomings of the traditional model-based adaptive controller. In [24], a novel robust decentralized control of electrically driven robot manipulator by adaptive fuzzy estimation and compensation uncertainty is proposed. The controller is designed via voltage control strategy. A fuzzy system is used to estimate and compensate uncertainty. In $[25,26]$, two adaptive PD control methods are investigated for trajectory tracking control of robot manipulators with known and unknown upper bound of the external disturbances, respectively. Both of the controllers are composed by a nonlinear PD feedback control law and an adaptive algorithm. The PD feedback control law can avoid large initial torque due to the large initial position error, and the adaptive algorithm can make good dynamic performance for the robot manipulator systems. However, the PD feedback control is rarely used in practical control systems. That is because the pure differential element cannot be realized in practice. Moreover, the differential action is very sensitive to system noise; as a result, if the PD control is adopted, any disturbances in each system's element would result in big fluctuation in systems output. Hence, the PD control is indeed of no benefit for the improvement of the system dynamic performance.

In the present study two new robust adaptive PID controllers are introduced for an $n$ degree-of-freedom robot manipulator systems with known or unknown upper bound of the external disturbances based on $[1,25,26]$. The designed controllers are composited by PID control and robust adaptive approach to cope with the external disturbances and unknown constant parameters that can arise. As regards the innovation of this study, an integration element is embedded in both PD control and robust adaptive algorithm based on the existing adaptive PD control laws $[25,26]$. With the adoption of the proposed controllers, the tolerable external disturbances are enlarged, and also the dynamic performance of the manipulator systems is improved and the finite-time tracking control accuracy is enhanced in contrast to those obtained with the usage of the adaptive PD controllers [25, 26]. By choosing adequate Lyapunov candidate functions and utilizing Barbalat's lemma, the system's closed-loop stability is proven. Some numerical results are also presented in order to demonstrate the control systems performance.

This paper is organized as follows. The nonlinear dynamics of rigid robot manipulator and some useful properties of dynamic systems are introduced in Section 2. In Section 3, three necessary assumptions for control laws development and systems stability analysis are given, and two robust adaptive PID controllers are designed for trajectory tracking control of robot manipulator with known or unknown upper bound of the external disturbances, respectively. The numerical verification of the controllers and the discussion are presented in Section 4. A short conclusion is given in Section 5.

\section{Dynamic Model of Robot Manipulator and Some Properties}

2.1. Dynamic Model. Generally, the dynamics of an $n$ degree of freedom $(n-\mathrm{DOF})$ rigid link robot manipulator with rotary joints can be expressed as [27]

$$
M(q) \ddot{q}+C(q, \dot{q}) \dot{q}+G(q)+u=\tau,
$$

where $q(t) \in R^{n}$ is the vector of joint angles, $M(q) \in R^{n \times n}$ is the inertia matrix of the manipulator, $C(q, \dot{q}) \in R^{n \times n}$ is the matrix of Coriolis and centripetal forces, $G(q) \in R^{n}$ is 


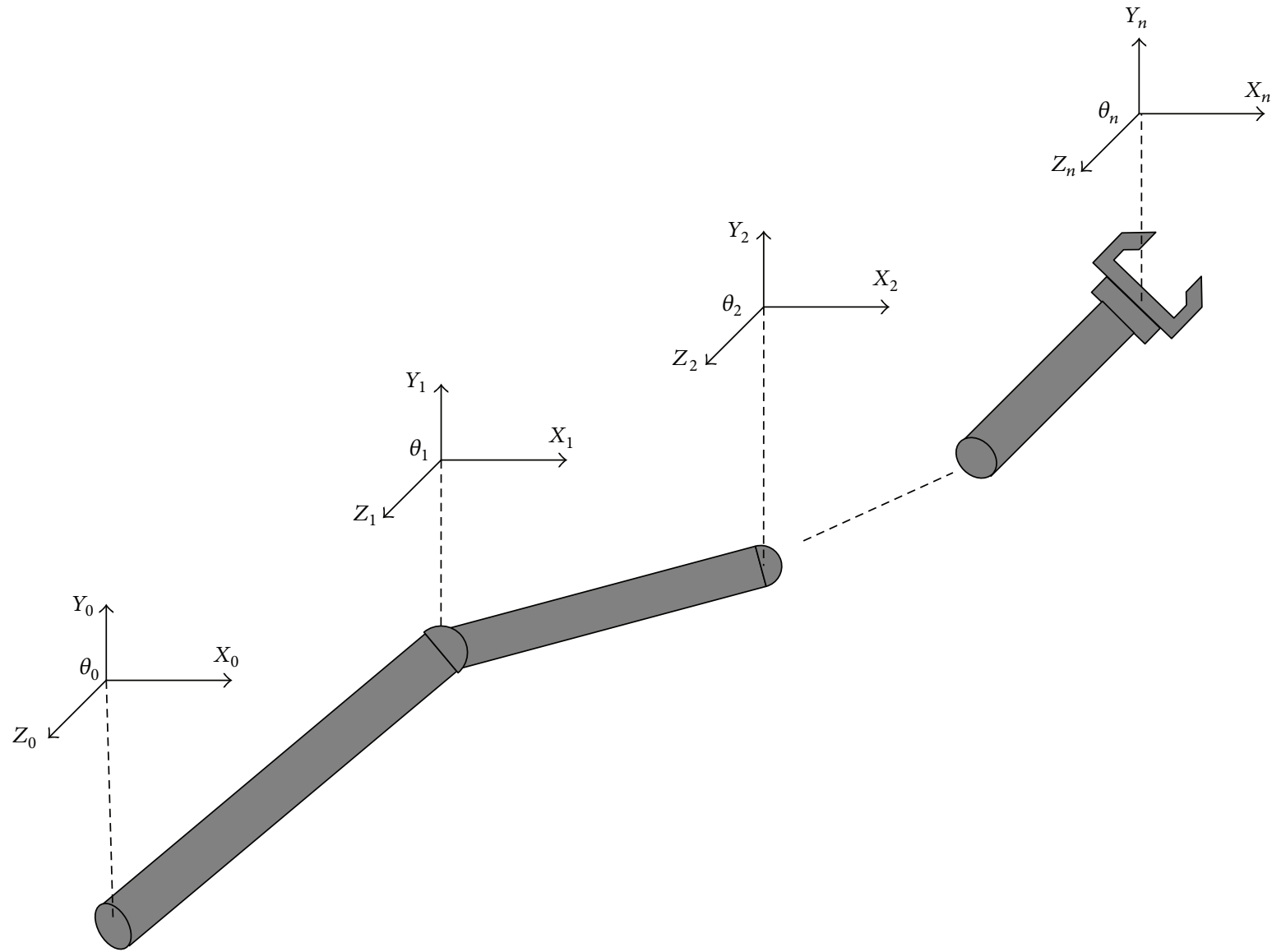

FIgURE 1: The frames assignment of an $n$-DOF rigid robot manipulator.

the vector of gravity factor, $\tau \in R^{n}$ is the vector of input torque, and $u \in R^{n}$ is the vector of all external disturbances.

Figure 1 presents the conceptual model of an $n$-DOF rigid robot manipulator. Assume that the manipulator is mounted on a fixed base, so the dynamic coupling between the manipulator and the base is neglected.

2.2. Dynamic System Properties. The dynamic systems given by (1) exhibit the following properties that are utilized in the subsequent control laws development and stability analysis [27].

(B1) The inertial matrix is symmetric and positive definite; that is, $M(q)=M^{T}(q)>0, \forall q \in R^{n}$. There are positive constants $m_{m}$ and $m_{M}$ such that $m_{m}\|y\|^{2} \leq$ $y^{T} M(q) y \leq m_{M}\|y\|^{2}, \forall y \in R^{n}$.

(B2) $\dot{M}(q)-2 C(q, \dot{q})$ is a skew-symmetric matrix; for example, $s^{T}[\dot{M}(q)-2 C(q, \dot{q})] s=0, \forall s \in R^{n}$.

(B3) $M(q), C(q, \dot{q})$, and $G(q)$ meet the linear condition of $M(q) \alpha+C(q, \dot{q}) \beta+G(q)=\Psi(q, \dot{q}, \alpha, \beta) P$, where $P \in$ $R^{m}$ is an unknown constant vector which describes the mass characteristics of the manipulator and $\Psi(q$, $\dot{q}, \alpha, \beta) \in R^{n \times m}$ is a known regression matrix.

\section{Robust Adaptive PID Control of Robot Manipulator}

Firstly, the following assumptions are imposed for the manipulator systems.

(C1) The desired trajectory $q_{d}$ and the time derivatives $\dot{q}_{d}$ and $\ddot{q}_{d}$ are available and bounded signals.

(C2) The external disturbances vector $u$ is bounded, and it is confined within the following limit:

$$
\|u\| \leq b_{1}+b_{2}\|e\|+b_{3}\|\dot{e}\|+b_{4}\left\|\int_{0}^{t} e d t\right\|,
$$

where $b_{1}, b_{2}, b_{3}$, and $b_{4}$ are positive constants, $e=$ $q-q_{d}$ and $\dot{e}=\dot{q}-\dot{q}_{d}$ are the position tracking error and the velocity tracking error, respectively, $\int_{0}^{t} e d t=$ $\left[\int_{0}^{t} e_{1} d t, \ldots, \int_{0}^{t} e_{n} d t\right]^{T}$.

(C3) $\ddot{e}(t)=\ddot{q}-\ddot{q}_{d}$ is existent and bounded in $t$.

Here we introduce two variables $x$ and $q_{k}$; meanwhile let

$$
\begin{gathered}
x=\dot{e}+\gamma e+\int_{0}^{t} e d t, \\
\dot{q}_{k}=\dot{q}_{d}-\gamma e-\int_{0}^{t} e d t,
\end{gathered}
$$

where the parameter $\gamma$ is a positive constant. 
With (3) giving

$$
\dot{q}-\dot{q}_{k}=x .
$$

With regard to the robot manipulator property (B3), let $\alpha=\ddot{q}_{k}, \beta=\dot{q}_{k}$; one obtains

$$
M(q) \ddot{q}_{k}+C(q, \dot{q}) \dot{q}_{k}+G(q)=\Psi\left(q, \dot{q}, \dot{q}_{k}, \ddot{q}_{k}\right) P .
$$

Substituting (4) into the above equation yields

$$
\begin{gathered}
M(q) \ddot{q}+C(q, \dot{q}) \dot{q}+G(q)-M(q) \dot{x}-C(q, \dot{q}) x \\
=\Psi\left(q, \dot{q}, \dot{q}_{k}, \ddot{q}_{k}\right) P .
\end{gathered}
$$

3.1. Robust Adaptive PID Controller Design with Known Upper Bound of the External Disturbances. For the robot manipulator systems (1), if the upper bound of the external disturbances signals $u$ is known, motivated by $[1,25]$, the controller which makes the position and the velocity tracking errors asymptotically converge to zero can be designed as follows:

$$
\begin{gathered}
\tau=-K_{P} e-K_{D} \dot{e}-K_{I}\left(\int_{0}^{t} e d t\right)+\Psi\left(q, \dot{q}, \dot{q}_{k}, \ddot{q}_{k}\right) \widehat{P}+V, \\
V=\left[v_{1}, \ldots, v_{n}\right]^{T} \\
v_{i}=-\left(b_{1}+b_{2}\|e\|+b_{3}\|\dot{e}\|+b_{4}\left\|\int_{0}^{t} e d t\right\|\right) \operatorname{sgn}\left(x_{i}\right),
\end{gathered}
$$

where $\widehat{P}$ is the estimate value of $P$.

Take the parameter estimation law of $\widehat{P}$ as

$$
\dot{\hat{P}}=-\Phi \Psi^{T}\left(q, \dot{q}, \dot{q}_{k}, \ddot{q}_{k}\right) x .
$$

The gain matrices are given by

$$
\begin{aligned}
K_{P} & =\operatorname{diag}\left[k_{P 1}, \ldots, k_{P n}\right], \\
K_{D} & =\operatorname{diag}\left[k_{D 1}, \ldots, k_{D n}\right], \\
K_{I} & =\operatorname{diag}\left[k_{I 1}, \ldots, k_{I n}\right],
\end{aligned}
$$

where $k_{P i}, k_{D i}, k_{I i}(i=1,2, \ldots, n)$ are all positive constants and $k_{D i}=k_{I i}, \Phi \in R^{m \times m}$ is a positive definite and symmetric matrix.

The framework of the proposed control scheme is shown in Figure 2.

Proof. Considering the Lyapunov function candidate,

$$
\begin{aligned}
V=\frac{1}{2}\left[x^{T} M(q) x+e^{T}\left(K_{P}+\gamma K_{D}\right) e\right. \\
\left.\quad+\left(\int_{0}^{t} e d t\right)^{T}\left(K_{P}+\gamma K_{I}\right)\left(\int_{0}^{t} e d t\right)+\widetilde{P}^{T} \Phi^{-1} \widetilde{P}\right]
\end{aligned}
$$

with $\widetilde{P}=\widehat{P}-P$.
From property (B1) one obtains

$$
\begin{aligned}
\left(x^{T} M(q) x\right)^{\prime} & =\dot{x}^{T} M(q) x+x^{T} \dot{M}(q) x+x^{T} M(q) \dot{x} \\
& =x^{T} \dot{M}(q) x+2 x^{T} M(q) \dot{x} .
\end{aligned}
$$

With the positive definite and symmetric matrices $K_{P}$, $K_{D}, K_{I}$, and $\Phi$, one gets

$$
\begin{gathered}
{\left[e^{T}\left(K_{P}+\gamma K_{D}\right) e\right]^{\prime}=2 e^{T}\left(K_{P}+\gamma K_{D}\right) \dot{e},} \\
{\left[\left(\int_{0}^{t} e d t\right)^{T}\left(K_{P}+\gamma K_{I}\right)\left(\int_{0}^{t} e d t\right)\right]^{\prime}} \\
=2\left(\int_{0}^{t} e d t\right)^{T}\left(K_{P}+\gamma K_{I}\right) e, \\
\left(\widetilde{P}^{T} \Phi^{-1} \widetilde{P}\right)^{\prime}=2 \widetilde{P}^{T} \Phi^{-1} \dot{\tilde{P}} .
\end{gathered}
$$

Therefore, one obtains

$$
\begin{aligned}
\dot{V}= & \frac{1}{2} x^{T} \dot{M}(q) x+x^{T} M(q) \dot{x} \\
& +e^{T}\left(K_{P}+\gamma K_{D}\right) \dot{e} \\
& +\left(\int_{0}^{t} e d t\right)^{T}\left(K_{P}+\gamma K_{I}\right) e+\widetilde{P}^{T} \Phi^{-1} \dot{\widetilde{P}} .
\end{aligned}
$$

Using (6) and (7) leads to

$$
\begin{aligned}
x^{T} M(q) \dot{x}= & x^{T}\left[\tau-u-\Psi\left(q, \dot{q}, \dot{q}_{k}, \ddot{q}_{k}\right) P-C(q, \dot{q}) x\right] \\
= & x^{T}\left[-K_{P} e-K_{D} \dot{e}-K_{I}\left(\int_{0}^{t} e d t\right)\right. \\
& \left.+\Psi\left(q, \dot{q}, \dot{q}_{k}, \ddot{q}_{k}\right) \widetilde{P}+V-u\right] \\
& -x^{T} C(q, \dot{q}) x .
\end{aligned}
$$

Considering $x^{T}=\dot{e}^{T}+\gamma e^{T}+\left(\int_{0}^{t} e d t\right)^{T}$, one gets

$$
\begin{aligned}
x^{T}( & \left.-K_{P} e-K_{D} \dot{e}-K_{I} \int_{0}^{t} e d t\right) \\
= & {\left[\dot{e}^{T}+\gamma e^{T}+\left(\int_{0}^{t} e d t\right)^{T}\right]\left[-K_{P} e-K_{D} \dot{e}-K_{I}\left(\int_{0}^{t} e d t\right)\right] } \\
= & -\dot{e}^{T} K_{P} e-\dot{e}^{T} K_{D} \dot{e}-\dot{e}^{T} K_{I}\left(\int_{0}^{t} e d t\right)-\gamma e^{T} K_{P} e \\
& -\gamma e^{T} K_{D} \dot{e}-\gamma e^{T} K_{I}\left(\int_{0}^{t} e d t\right)-\left(\int_{0}^{t} e d t\right)^{T} K_{P} e \\
& -\left(\int_{0}^{t} e d t\right)^{T} K_{D} \dot{e}-\left(\int_{0}^{t} e d t\right)^{T} K_{I}\left(\int_{0}^{t} e d t\right) .
\end{aligned}
$$




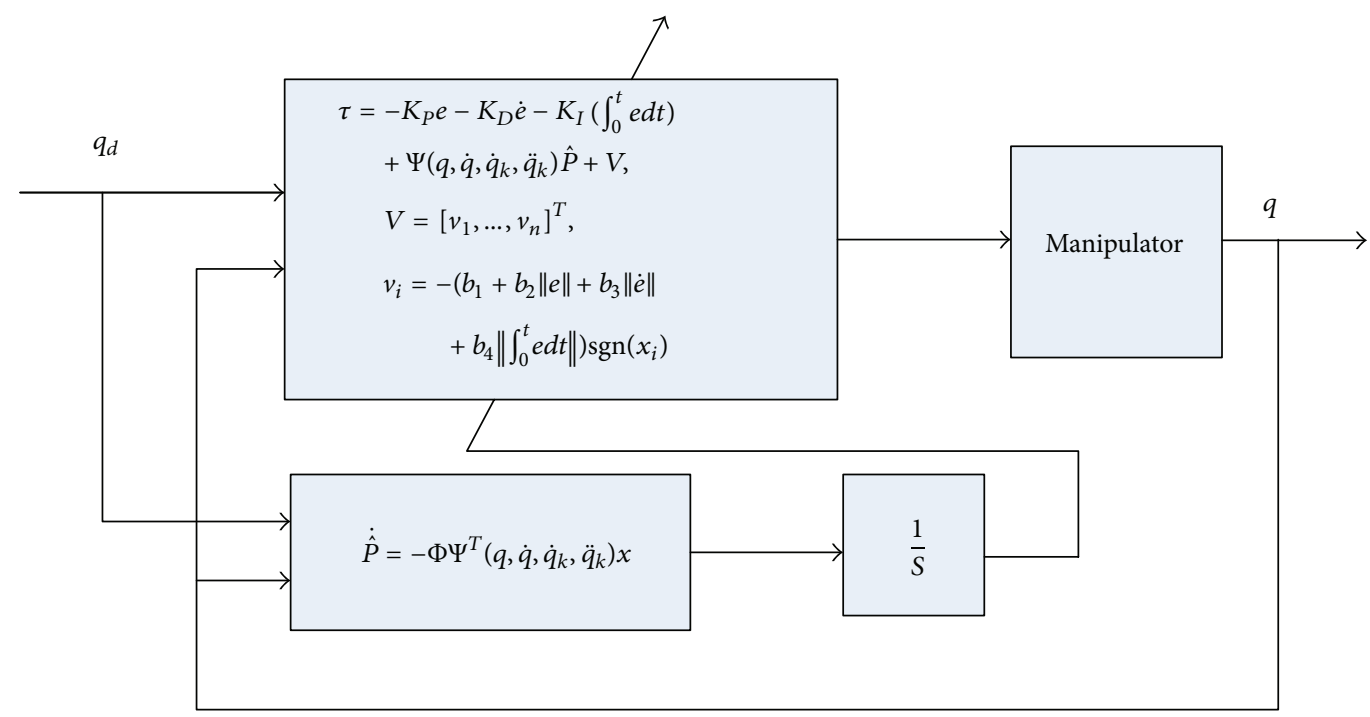

FIGURE 2: Framework of control law with known upper bound of the disturbances.

Substituting (16) and (17) into (15) yields

$$
\begin{aligned}
\dot{V}= & -\dot{e}^{T} K_{D} \dot{e}-\gamma e^{T} K_{P} e-\left(\int_{0}^{t} e d t\right)^{T} K_{I}\left(\int_{0}^{t} e d t\right) \\
& -\dot{e}^{T}\left(K_{D}+K_{I}\right)\left(\int_{0}^{t} e d t\right)+\frac{1}{2} x^{T} \dot{M}(q) x-x^{T} C(q, \dot{q}) x \\
& +\widetilde{P}^{T} \Phi^{-1} \dot{\widetilde{P}}+x^{T} \Psi\left(q, \dot{q}, \dot{q}_{k}, \ddot{q}_{k}\right) \widetilde{P}+x^{T}(V-u) .
\end{aligned}
$$

With property (B2) one obtains

$\frac{1}{2} x^{T} \dot{M}(q) x-x^{T} C(q, \dot{q}) x=\frac{1}{2} x^{T}[\dot{M}(q)-2 C(q, \dot{q})] x=0$.

Note that

$x^{T} \Psi\left(q, \dot{q}, \dot{q}_{k}, \ddot{q}_{k}\right) \widetilde{P}=\widetilde{P}^{T} \Psi^{T}\left(q, \dot{q}, \dot{q}_{k}, \ddot{q}_{k}\right) x, \quad \dot{\widetilde{P}}=\dot{\widehat{P}}$,

and one gets

$$
\begin{aligned}
\widetilde{P}^{T} \Phi^{-1} \dot{\widetilde{P}}+x^{T} \Psi\left(q, \dot{q}, \dot{q}_{k}, \ddot{q}_{k}\right) \widetilde{P} \\
=\widetilde{P}^{T} \Phi^{-1}\left[-\Phi \Psi^{T}\left(q, \dot{q}, \dot{q}_{k}, \ddot{q}_{k}\right) x\right] \\
\quad+\widetilde{P}^{T} \Psi^{T}\left(q, \dot{q}, \dot{q}_{k}, \ddot{q}_{k}\right) x=0 .
\end{aligned}
$$

Thus, one obtains

$$
\begin{aligned}
\dot{V}= & -\dot{e}^{T} K_{D} \dot{e}-\gamma e^{T} K_{P} e-\left(\int_{0}^{t} e d t\right)^{T} K_{I}\left(\int_{0}^{t} e d t\right) \\
& -\dot{e}^{T}\left(K_{D}+K_{I}\right)\left(\int_{0}^{t} e d t\right)+x^{T}(V-u) .
\end{aligned}
$$

Note that the following equalities and inequalities hold

$$
\begin{gathered}
\dot{e}^{T} K_{D} \dot{e}=\sum_{i=1}^{n} k_{D i} \dot{e}_{i}^{2}, \quad \gamma e^{T} K_{P} e=\sum_{i=1}^{n} \gamma k_{P i} e_{i}^{2}, \\
\left(\int_{0}^{t} e d t\right)^{T} K_{I}\left(\int_{0}^{t} e d t\right)=\sum_{i=1}^{n} k_{I i}\left(\int_{0}^{t} e_{i} d t\right)^{2}, \\
\dot{e}^{T}\left(K_{D}+K_{I}\right)\left(\int_{0}^{t} e d t\right)= \\
\sum_{i=1}^{n}\left(k_{D i}+k_{I i}\right) \dot{e}_{i}\left(\int_{0}^{t} e_{i} d t\right), \\
\leq \\
-\frac{1}{2} \sum_{i=1}^{n}\left(k_{D i}+k_{I i}\right) \dot{e}_{i}^{2} \\
+\frac{1}{2} \sum_{i=1}^{n}\left(k_{D i}+k_{I i}\right)\left(\int_{0}^{t} e_{i}\left(\int_{0}^{t} e_{i} d t\right)\right.
\end{gathered}
$$

Hence, one gets

$$
\begin{aligned}
\dot{V}= & -\sum_{i=1}^{n} k_{D i} \dot{e}_{i}^{2}-\sum_{i=1}^{n} \gamma k_{P i} e_{i}^{2}-\sum_{i=1}^{n} k_{I i}\left(\int_{0}^{t} e_{i} d t\right)^{2} \\
& -\sum_{i=1}^{n}\left(k_{D i}+k_{I i}\right) \dot{e}_{i}\left(\int_{0}^{t} e_{i} d t\right)+x^{T}(V-u) \\
\leq & -\sum_{i=1}^{n} k_{D i} \dot{e}_{i}^{2}-\sum_{i=1}^{n} \gamma k_{P i} e_{i}^{2}-\sum_{i=1}^{n} k_{I i}\left(\int_{0}^{t} e_{i} d t\right)^{2} \\
& +\frac{1}{2} \sum_{i=1}^{n}\left(k_{D i}+k_{I i}\right) \dot{e}_{i}^{2}+\frac{1}{2} \sum_{i=1}^{n}\left(k_{D i}+k_{I i}\right)\left(\int_{0}^{t} e_{i} d t\right)^{2} \\
& +x^{T}(V-u)
\end{aligned}
$$




$$
\begin{aligned}
\leq & -\sum_{i=1}^{n}\left[k_{D i}-\frac{1}{2}\left(k_{D i}+k_{I i}\right)\right] \dot{e}_{i}^{2}-\sum_{i=1}^{n} \gamma k_{P i} e_{i}^{2} \\
& -\sum_{i=1}^{n}\left[k_{I i}-\frac{1}{2}\left(k_{D i}+k_{I i}\right)\right]\left(\int_{0}^{t} e_{i} d t\right)^{2}+x^{T}(V-u) .
\end{aligned}
$$

Now considering the term of $x^{T}(V-u)$,

$$
\begin{aligned}
x^{T} V & =\sum_{i=1}^{n} x_{i}\left[-\left(b_{1}+b_{2}\|e\|+b_{3}\|\dot{e}\|+b_{4}\left\|\int_{0}^{t} e d t\right\|\right) \operatorname{sgn}\left(x_{i}\right)\right] \\
& =\sum_{i=1}^{n}\left[-\left(b_{1}+b_{2}\|e\|+b_{3}\|\dot{e}\|+b_{4}\left\|\int_{0}^{t} e d t\right\|\right)\left|x_{i}\right|\right] \\
& \leq \sum_{i=1}^{n}\left(-\|u\| \cdot\left|x_{i}\right|\right) ;
\end{aligned}
$$

here the assumption (C2) has been used.

Note that

$$
-x^{T} u \leq\left\|x^{T}\right\| \cdot\|u\|
$$

Defining $\left\|x^{T}\right\|=\sum_{i=1}^{n}\left|x_{i}\right|$ gives

$$
\begin{aligned}
\dot{V} \leq & -\sum_{i=1}^{n}\left[k_{D i}-\frac{1}{2}\left(k_{D i}+k_{I i}\right)\right] \dot{e}_{i}^{2}-\sum_{i=1}^{n} \gamma k_{P i} e_{i}^{2} \\
& -\sum_{i=1}^{n}\left[k_{I i}-\frac{1}{2}\left(k_{D i}+k_{I i}\right)\right]\left(\int_{0}^{t} e_{i} d t\right)^{2} \\
& +\sum_{i=1}^{n}\left(-\|u\| \cdot\left|x_{i}\right|\right)+\left\|x^{T}\right\| \cdot\|u\| \\
\leq & -\sum_{i=1}^{n}\left[k_{D i}-\frac{1}{2}\left(k_{D i}+k_{I i}\right)\right] \dot{e}_{i}^{2}-\sum_{i=1}^{n} \gamma k_{P i} e_{i}^{2} \\
& -\sum_{i=1}^{n}\left[k_{I i}-\frac{1}{2}\left(k_{D i}+k_{I i}\right)\right]\left(\int_{0}^{t} e_{i} d t\right)^{2} .
\end{aligned}
$$

With $k_{D i}=k_{I i}>0$ and $k_{P i}>0$ one obtains

$$
\begin{gathered}
k_{D i}-\frac{1}{2}\left(k_{D i}+k_{I i}\right)=0, \quad \gamma k_{P i}>0, \\
k_{I i}-\frac{1}{2}\left(k_{D i}+k_{I i}\right)=0 .
\end{gathered}
$$

Finally, one gets

$$
\dot{V} \leq-\sum_{i=1}^{n} \gamma k_{P i} e_{i}^{2}
$$

From the proof and analysis above we know that the function $\dot{V}$ is negative and vanishes if and only if $e(t)=0$; thus the position tracking error goes to zero as time goes to infinity; namely, $\lim _{t \rightarrow \infty} e(t)=0$.
According to the assumption (C3) we obtain that $\dot{e}(t)$ : $R^{+} \rightarrow R$ is uniformly continuous [28]. Consider the following formula holds:

$$
\begin{aligned}
\lim _{t \rightarrow \infty} \int_{0}^{t} \dot{e}(\tau) d \tau & =\lim _{t \rightarrow \infty}[e(t)-e(0)]=\lim _{t \rightarrow \infty} e(t)-e(0) \\
& =-e(0)<\infty
\end{aligned}
$$

which implies that the limit $\lim _{t \rightarrow \infty} \int_{0}^{t} \dot{e}(\tau) d \tau$ is existent and bounded. Therefore, it follows from the Barbalat's lemma [29] that $\dot{e}(t) \rightarrow 0$ as $t \rightarrow \infty$; that is, $\lim _{t \rightarrow \infty} \dot{e}(t)=0$.

Hence, the designed controller can guarantee the equilibrium $(e, \dot{e})=(0,0)$ globally asymptotically stable. It is also seen that the parameter vector $\widetilde{P}$ is bounded but does not necessarily converge to zero.

\subsection{Robust Adaptive PID Controller Design with Unknown} Upper Bound of the External Disturbances. On the other hand, if the upper bound of the external disturbances signals $u$ is unknown, inspired by $[1,26]$, the controller which ensures the global asymptotical stability of the manipulator positions and velocities can be designed as follows:

$$
\tau=-K_{P} e-K_{D} \dot{e}-K_{I}\left(\int_{0}^{t} e d t\right)+\Psi\left(q, \dot{q}, \dot{q}_{k}, \ddot{q}_{k}\right) \widehat{P}+V
$$

$$
\begin{gathered}
V=-\frac{(\widehat{b} \xi)^{2}}{(\widehat{b} \xi)\|x\|+\varepsilon^{2}} \cdot x, \\
\dot{\vec{b}}=\lambda_{1} \xi\|x\|, \quad \widehat{b}(0)=0, \\
\dot{\varepsilon}=-\lambda_{2} \varepsilon, \quad \varepsilon(0) \neq 0,
\end{gathered}
$$

where $b=b_{1}+b_{2}+b_{3}+b_{4}, \tilde{b}=b-\widehat{b}, \widehat{b}$ is the estimate value of $b, \xi=\max \left(1,\|e\|,\|\dot{e}\|,\left\|\int_{0}^{t} e d t\right\|\right), \lambda_{1}$ and $\lambda_{2}$ are the arbitrary positive constants, and other parameters are defined as Section 3.1.

The framework of the proposed control scheme is shown in Figure 3.

Proof. Consider the following Lyapunov function:

$$
\begin{aligned}
V=\frac{1}{2}\left[x^{T}\right. & M(q) x+e^{T}\left(K_{P}+\gamma K_{D}\right) e \\
& +\left(\int_{0}^{t} e d t\right)^{T}\left(K_{P}+\gamma K_{I}\right)\left(\int_{0}^{t} e d t\right) \\
& \left.+\widetilde{P}^{T} \Phi^{-1} \widetilde{P}+\left(\lambda_{1}^{-1} \widetilde{b}^{2}+\lambda_{2}^{-1} \varepsilon^{2}\right)\right] .
\end{aligned}
$$

Based on the controller stability analysis with known upper bound of the external disturbances in Section 3.1, according to (24) one gets

$$
\dot{V} \leq-\sum_{i=1}^{n} \gamma k_{P i} e_{i}^{2}+x^{T} V-x^{T} u+\lambda_{1}^{-1} \tilde{b} \dot{\vec{b}}+\lambda_{2}^{-1} \varepsilon \dot{\varepsilon} .
$$




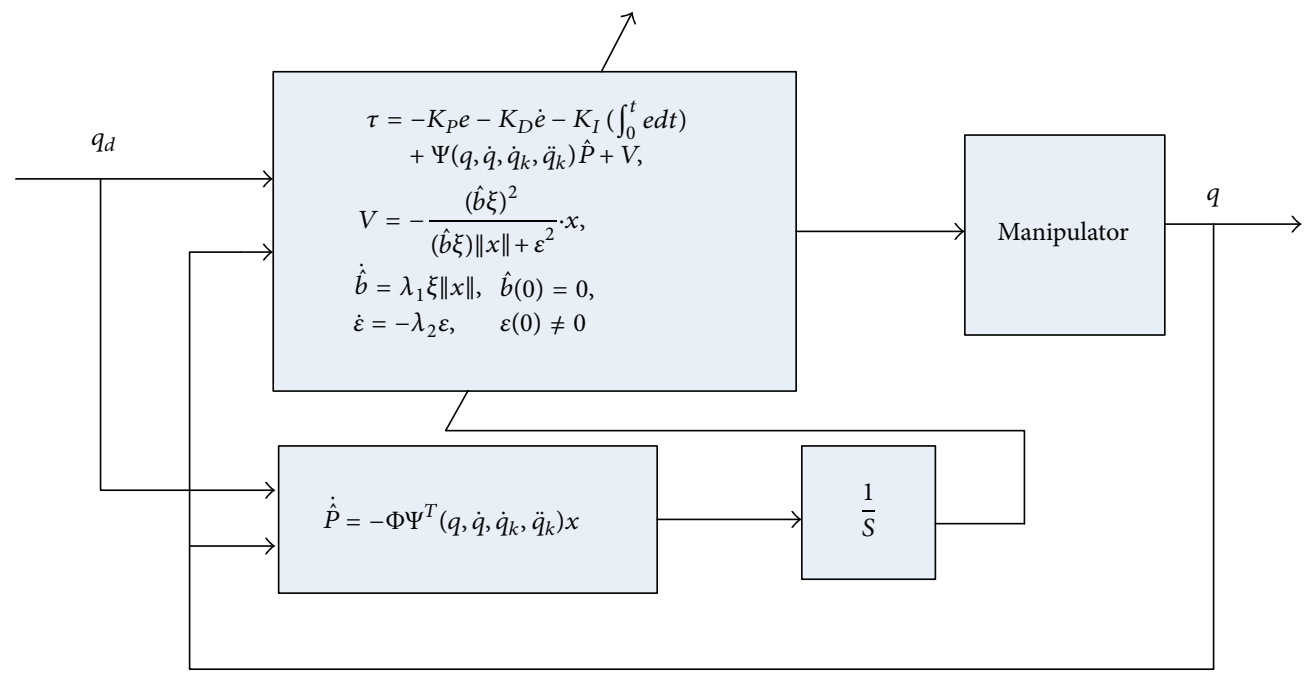

FIGURE 3: Framework of control law with unknown upper bound of the disturbances.

Substituting (32) and (34) into (36) yields

$\dot{V} \leq-\sum_{i=1}^{n} \gamma k_{P i} e_{i}^{2}+x^{T}\left[-\frac{(\widehat{b} \xi)^{2}}{(\widehat{b} \xi)\|x\|+\varepsilon^{2}}\right] x-x^{T} u+\lambda_{1}^{-1} \widetilde{b} \dot{\vec{b}}-\varepsilon^{2}$.

Take into account that the following equalities and inequalities hold:

$$
\begin{gathered}
x^{T} x=\|x\|^{2}, \quad-x^{T} u \leq\|x\| \cdot\|u\|, \\
\|u\| \leq b_{1}+b_{2}\|e\|+b_{3}\|\dot{e}\|+b_{4}\left\|\int_{0}^{t} e d t\right\| \leq b \xi, \\
\dot{\vec{b}}=-\dot{\vec{b}}=-\lambda_{1} \xi\|x\| ;
\end{gathered}
$$

here the assumption (C2) and (33) have been used.

Therefore, one obtains

$$
\begin{aligned}
\dot{V} \leq & -\sum_{i=1}^{n} \gamma k_{P i} e_{i}^{2} \\
& -\frac{(\widehat{b} \xi)^{2}}{(\widehat{b} \xi)\|x\|+\varepsilon^{2}} \cdot\|x\|^{2}+\|x\| \cdot(b \xi)-\widetilde{b} \xi\|x\|-\varepsilon^{2} \\
\leq & -\sum_{i=1}^{n} \gamma k_{P i} e_{i}^{2} \\
& +\frac{-(\widehat{b} \xi)^{2}\|x\|^{2}+(\widehat{b} \xi)^{2}\|x\|^{2}+\varepsilon^{2} \xi\|x\| \widehat{b}-\varepsilon^{2}(\widehat{b} \xi)\|x\|-\varepsilon^{4}}{(\widehat{b} \xi)\|x\|+\varepsilon^{2}} \\
\leq & -\sum_{i=1}^{n} \gamma k_{P i} e_{i}^{2}-\frac{\varepsilon^{4}}{(\widehat{b} \xi)\|x\|+\varepsilon^{2}} .
\end{aligned}
$$

From the definitions of (33) and (34), it can be easily verified that $\widehat{b}>0$ and $\varepsilon^{2}>0$ as $t>0$, and $\varepsilon \rightarrow 0$ as $t \rightarrow \infty$. Thus the condition $\dot{V}<0$ is satisfied. According to the Lyapunov stability theory and Barbalat's lemma (see (30)), the convergence of $e$ and $\dot{e}$ to zero is guaranteed, and also the boundedness of the parameter vector $\widetilde{P}$ is obtained.

\section{Simulation Experiments and Discussion}

In order to illustrate the performance of the proposed robust adaptive PID controllers, simulation results are given by means of MATLAB/SIMULINK. A comparison between the adaptive PD controllers in $[25,26]$ and the robust adaptive PID controllers derived in this study is carried out. The simulation model is a two-degree-of-freedom planar rigid manipulator with rotary joints. According to (1), its dynamic equation can be described as follows [30]:

$$
\begin{gathered}
{\left[\begin{array}{ll}
M_{11}\left(q_{2}\right) & M_{12}\left(q_{2}\right) \\
M_{12}\left(q_{2}\right) & M_{22}\left(q_{2}\right)
\end{array}\right]\left[\begin{array}{l}
\ddot{q}_{1} \\
\ddot{q}_{2}
\end{array}\right]} \\
+\left[\begin{array}{ll}
-C_{12}\left(q_{2}\right) \dot{q}_{2} & -C_{12}\left(q_{2}\right)\left(\dot{q}_{1}+\dot{q}_{2}\right) \\
C_{12}\left(q_{2}\right) \dot{q}_{1} & 0
\end{array}\right] \\
+\left[\begin{array}{l}
\dot{q}_{1} \\
\dot{q}_{2}
\end{array}\right]+\left[\begin{array}{l}
G_{1}\left(q_{1}, q_{2}\right) g \\
G_{2}\left(q_{1}, q_{2}\right) g
\end{array}\right]=\left[\begin{array}{l}
\tau_{1} \\
\tau_{2}
\end{array}\right],
\end{gathered}
$$

where

$$
\begin{gathered}
M_{11}\left(q_{2}\right)=\left(m_{1}+m_{2}\right) r_{1}^{2}+m_{2} r_{2}^{2}+2 m_{2} r_{1} r_{2} \cos q_{2}, \\
M_{12}\left(q_{2}\right)=m_{2} r_{2}^{2}+m_{2} r_{1} r_{2} \cos q_{2}, \\
M_{22}\left(q_{2}\right)=m_{2} r_{2}^{2}, \\
C_{12}\left(q_{2}\right)=m_{2} r_{1} r_{2} \sin q_{2}, \\
G_{1}\left(q_{1}, q_{2}\right)=\left(m_{1}+m_{2}\right) r_{1} \cos q_{2}+m_{2} r_{2} \cos \left(q_{1}+q_{2}\right), \\
G_{2}\left(q_{1}, q_{2}\right)=m_{2} r_{2} \cos \left(q_{1}+q_{2}\right) .
\end{gathered}
$$

At first, linearize (40) and give the parameter matrices $\Psi$ and $P$. 
According to (5) the following equality holds:

$M(q) \ddot{q}_{k}+C(q, \dot{q}) \dot{q}_{k}+G(q)=\Psi\left(q, \dot{q}, \dot{q}_{k}, \ddot{q}_{k}\right) P$.

Let the matrices $\Psi$ and $P$ have the following forms:

$$
\Psi=\left[\begin{array}{lll}
\psi_{11} & \psi_{12} & \psi_{13} \\
\psi_{21} & \psi_{22} & \psi_{23}
\end{array}\right], \quad P=\left[\begin{array}{lll}
p_{1} & p_{2} & p_{3}
\end{array}\right]^{T} .
$$

Then the parameters can be derived as below:

$$
\begin{gathered}
\psi_{11}=\ddot{q}_{1 k}+\left(\frac{g}{r_{1}}\right) \cos q_{2}, \quad \psi_{12}=\ddot{q}_{1 k}+\ddot{q}_{2 k}, \\
\psi_{13}=2 \ddot{q}_{1 k} \cos q_{2}+\ddot{q}_{2 k} \cos q_{2}-\dot{q}_{2} \dot{q}_{1 k} \sin q_{2} \\
-\left(\dot{q}_{1}+\dot{q}_{2}\right) \dot{q}_{2 k} \sin q_{2}+\left(\frac{g}{r_{1}}\right) \cos \left(q_{1}+q_{2}\right), \\
\psi_{21}=0, \quad \psi_{22}=\psi_{12}, \\
\psi_{23}=\dot{q}_{1} \dot{q}_{1 k} \sin q_{2}+\ddot{q}_{1 k} \cos q_{2}+\left(\frac{g}{r_{1}}\right) \cos \left(q_{1}+q_{2}\right), \\
p_{1}=\left(m_{1}+m_{2}\right) r_{1}^{2}, \quad p_{2}=m_{2} r_{2}^{2}, \quad p_{3}=m_{2} r_{1} r_{2} .
\end{gathered}
$$

The link parameters of robot manipulator are given by $r_{1}=1 \mathrm{~m}, r_{2}=0.8 \mathrm{~m}, m_{1}=0.5 \mathrm{~kg}$, and $m_{2}=0.5 \mathrm{~kg}$. The upper bound parameters are selected as $b_{1}=2, b_{2}=3, b_{3}=6$, and $b_{4}=1$. The disturbances vector is chosen as $u=\left[\begin{array}{ll}1.0 & 1.0\end{array}\right]^{T}+$ $2 e+5 \dot{e}$. The reference trajectories are $q_{1 d}=\cos (\pi t)$ and $q_{2 d}=\cos (\pi t)$. The initial values of manipulator positions and velocities are selected as $\left[\begin{array}{llll}q_{1} & \dot{q}_{1} & q_{2} & \dot{q}_{2}\end{array}\right]^{T}=\left[\begin{array}{llll}0.6 & 0 & 0.3 & 0\end{array}\right]^{T}$.

Take the gain coefficients of the robust adaptive PID control as

$$
\begin{aligned}
& K_{P}=\operatorname{diag}[1000,400], \quad K_{D}=\operatorname{diag}[180,150], \\
& K_{I}=K_{D}, \quad \gamma=5, \quad \Phi=\operatorname{diag}[5.0,5.0,5.0] .
\end{aligned}
$$

The adaptive PD control gains $K_{P}$ and $K_{D}$ are determined by $[25,26]$.

The MATLAB/SIMULINK framework is shown in Figure 4.

In order to show the advantage of the proposed control laws, two simulations are conducted, one with adaptive PD control and the other with robust adaptive PID control. The simulation results are presented in Figures 5-16.

4.1. Simulation Results with Known Upper Bound of the External Disturbances. Adopting the control law described in Section 3.1 to simulate the tracking effects of robot manipulator, the simulation results can be seen from Figures 5, 6, 7, 8,9 , and 10 .

4.2. Simulation Results with Unknown Upper Bound of the External Disturbances. Choosing $\lambda_{1}=\lambda_{2}=40$ and utilizing the control scheme represented in Section 3.2 to express the tracking capability of robot manipulator, the simulation results are shown in Figures 11, 12, 13, 14, 15, and 16.

\section{Discussion}

These figures indicate that the robust adaptive PID control schemes can compensate the unknown bounded external disturbances and guarantee the manipulator systems to track the desired position and velocity trajectories accurately with quite small tracking errors in finite time. The adaptive algorithm can effectively estimate the unknown constant vector $P$ which describes the mass characteristics of robot manipulator.

In comparison with the adaptive $\mathrm{PD}$ controllers proposed in $[25,26]$, the robust adaptive PID controllers can provide better control performance. The main reason lies in the incorporation of an integral action within both PD control and robust adaptive algorithm based on the adaptive PD control laws $[25,26]$. Concerning the PID control term in the robust adaptive PID controllers, it exhibits the superiority in contrast to the PD one in the adaptive PD controllers $[25,26]$. In the case of PD control, the differential element only plays a part in dynamic process, and it has no effect on steadystate process, although the steady-state errors can be smaller by increasing the proportional gain matrix; yet, too large proportional gain may cause the system to be unstable. In addition, from a practical point of view, the pure differential element cannot be realized in practice; furthermore, the differential action is very sensitive to system noise; as a result, any disturbances in each system's element would result in big fluctuation in systems output. Hence, the adoption of PD control is indeed of no benefit for the improvement of system dynamic performance. While the PID control includes an integral element which can raise the indiscrimination degree of the system, so it can enhance the stead-state performance of the closed-loop system. Apart from that, the PID control can also provide one negative real zero, which contributes to improve the dynamic performance of robot manipulator systems. In terms of the adaptation algorithm in this work, different from the one in $[25,26]$, an integral element acts on the estimation law $\dot{\hat{P}}$ via the variable $x$ (see (3) and (10)). In this case, note that continuously accumulated position errors under the action of integration element result in a bigger value of $\dot{\hat{P}}$ than that in $[25,26]$, which implies that the estimated rate of the estimate vector $\widehat{P}$ is increased. As a consequence, the dynamic performance and the finite-time estimation accuracy of the estimate vector $\widehat{P}$ are improved with comparison to those in $[25,26]$. In addition to these, note that assumption (C2) provides a bigger upper bound of the external disturbances than the one which is defined as $\|u\| \leq b_{1}+b_{2}\|e\|+b_{3}\|\dot{e}\|$ in $[25,26]$. Because when the two upper bound inequalities of the external disturbances signals $u$ in $[25,26]$ and this study hold the same parameter values of $b_{1}, b_{2}$, and $b_{3}$, an arbitrary positive constant $b_{4}$ together with the nonzero accumulated position errors in assumption (C2) can lead to a bigger upper bound value for the external disturbances signals $u$. However, the enlarged external disturbances can be compensated by the enhanced robust term $V$ (see (3), (9), (32), and (33)). Therefore, greater external disturbances are allowed for this study than the ones tolerated in $[25,26]$. Hence, the proposed robust adaptive PID 


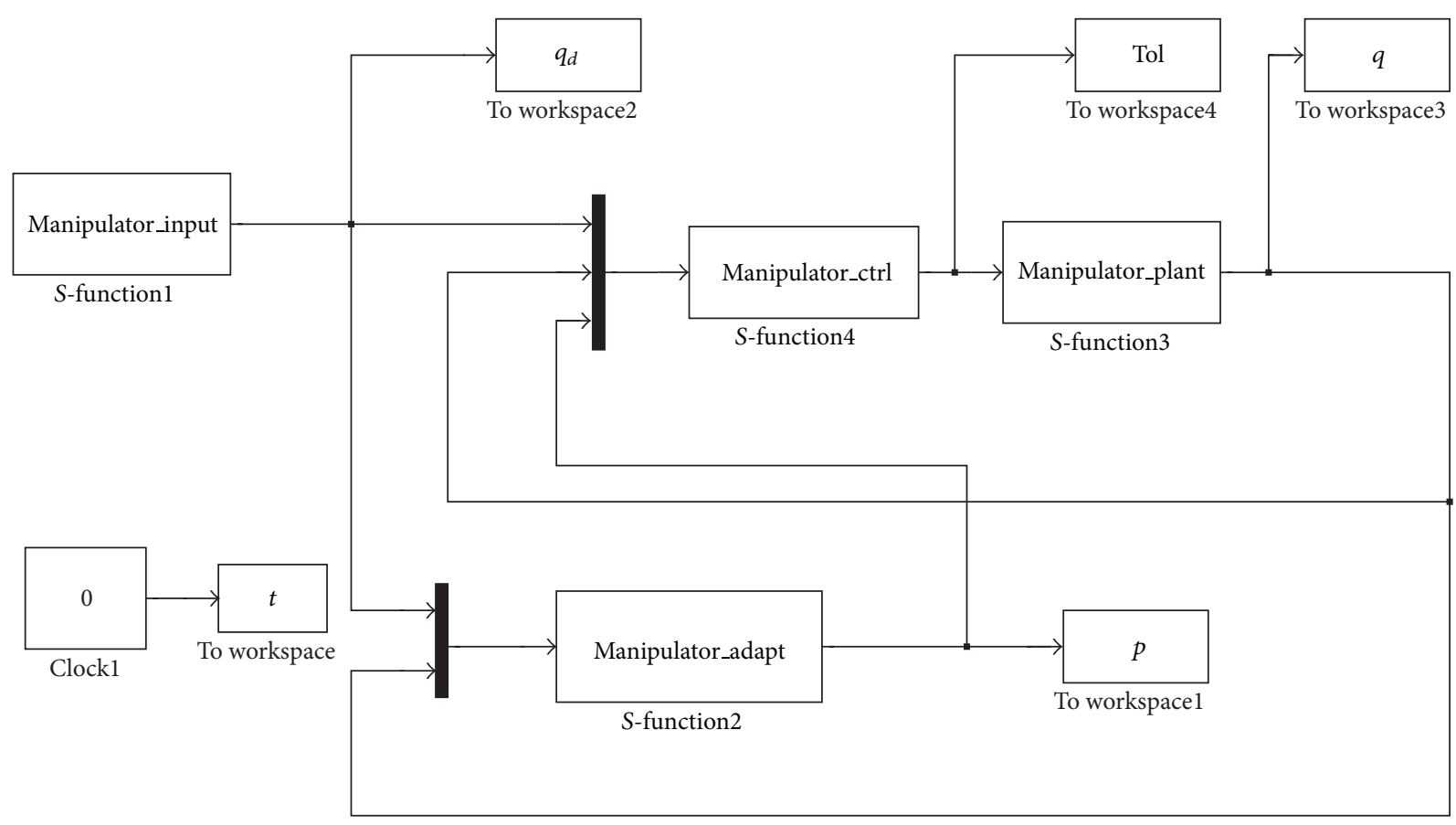

Figure 4: The MATLAB/SIMULINK framework.

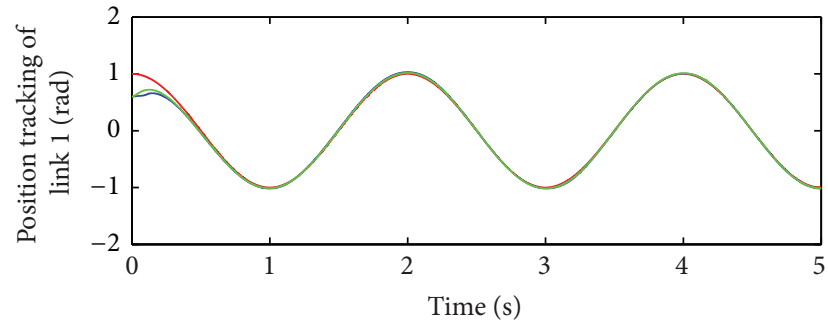

(a)

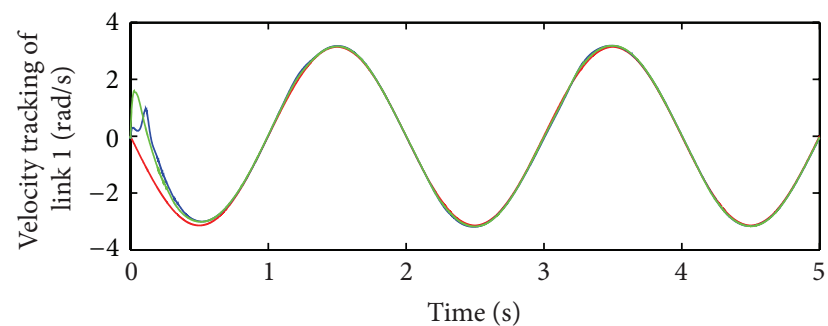

- Desired trajectory

- Actual trajectory of adaptive PD

- Actual trajectory of adaptive PID

(b)

FIGURE 5: Position and velocity trajectory tracking of link 1.

control laws could show their advantages with appropriate control gains in contrast to the adaptive PD ones $[25,26]$.

However, it should emphasize that the proposed robust adaptive PID controllers can only enhance the finite-time tracking accuracy in contrast to the existing adaptive PD ones $[25,26]$, because the global asymptotical stability with

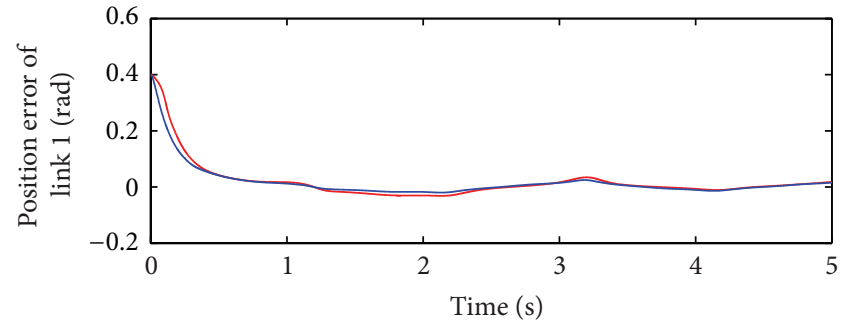

(a)

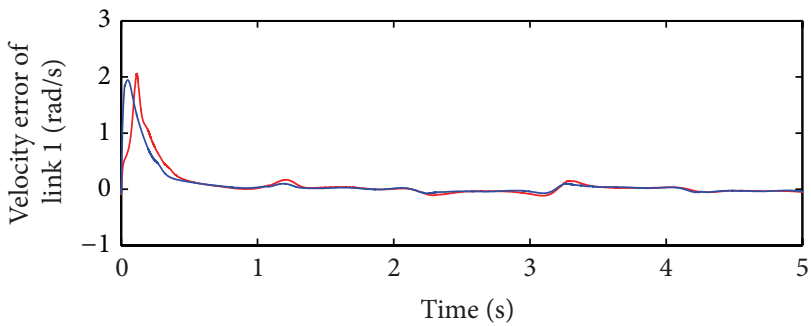

— Tracking error of adaptive PD

_ Tracking error of adaptive PID

(b)

FIgURE 6: Position and velocity trajectory tracking errors of link 1.

respect to the manipulator positions and velocities can also be achieved by using the adaptive PD control laws $[25,26]$, which implies that $e(t) \rightarrow 0$ and $\dot{e}(t) \rightarrow 0$ as $t \rightarrow \infty$ can be guaranteed. Nevertheless, the dynamic performance of the closed-loop system can be improved in the whole process by adopting the robust adaptive PID control laws. In fact, the control procedure is always the finite-time process in 


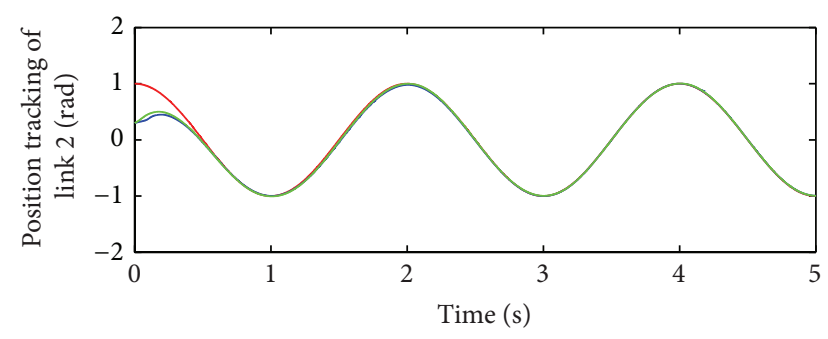

(a)

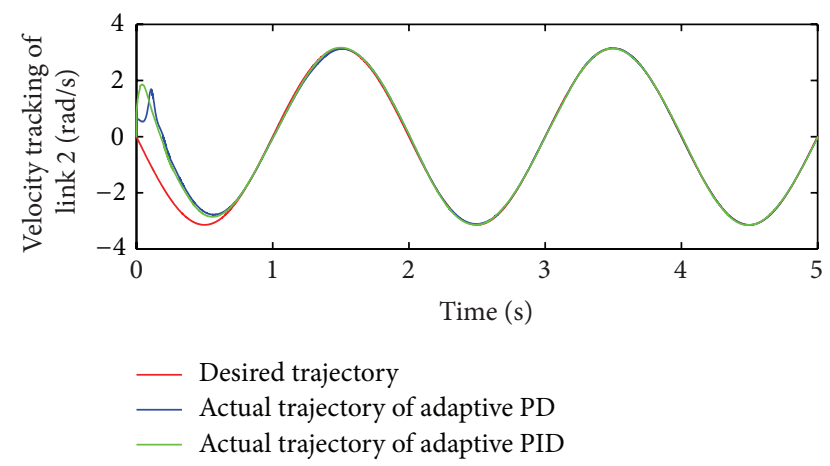

(b)

FIGURE 7: Position and velocity trajectory tracking of link 2.

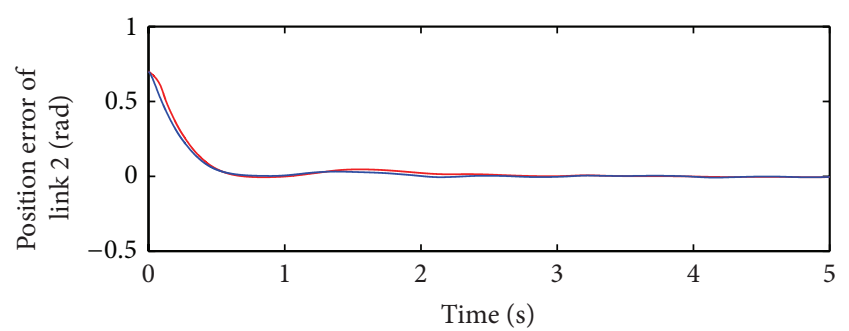

(a)

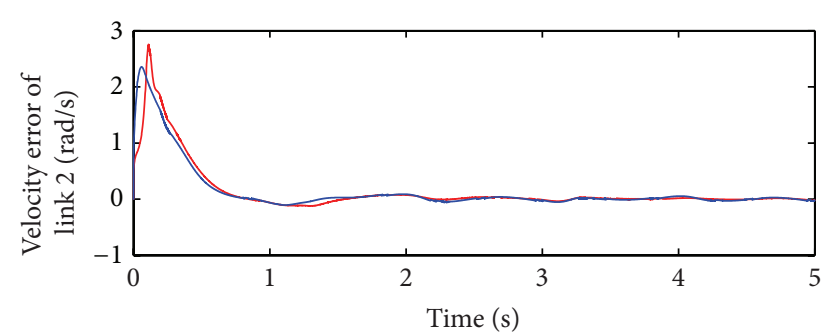

_ Tracking error of adaptive PD

Tracking error of adaptive PID

(b)

FIGURE 8: Position and velocity trajectory tracking errors of link 2.

practical control systems; hence the study of the finite-time control performance improvement of robot manipulator in this work is of practical significance.

Simulation results verify the advantages of the proposed control schemes. The experimental curves show that the robust adaptive PID controllers provide higher accuracy in finite-time position and velocity tracking control than those

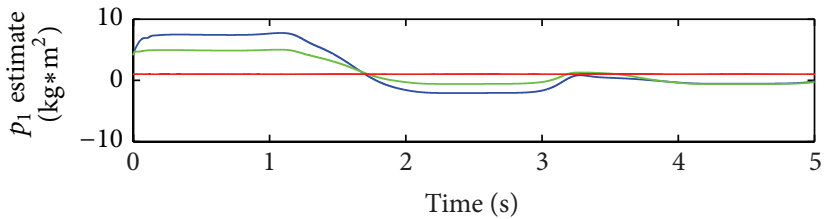

(a)

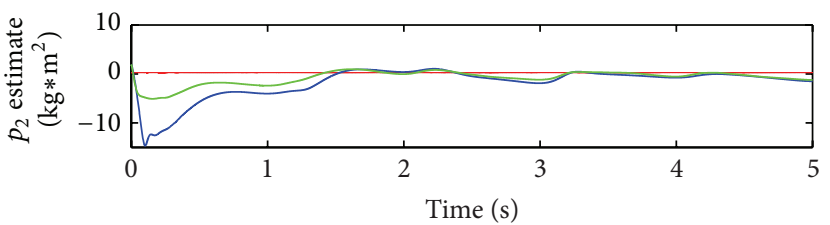

(b)

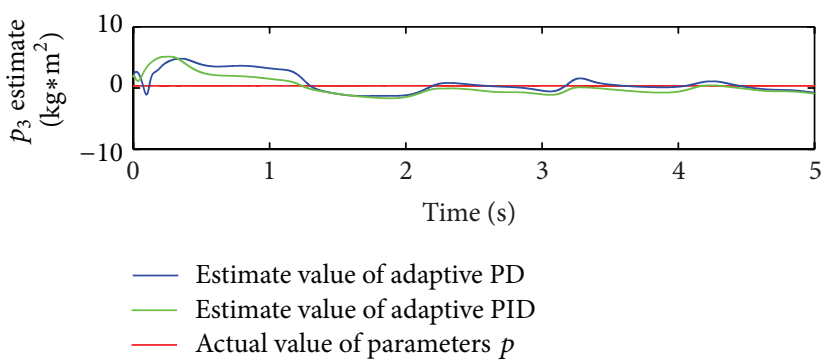

(c)

FIGURE 9: Estimate values of parameters $p_{1}, p_{2}$, and $p_{3}$.

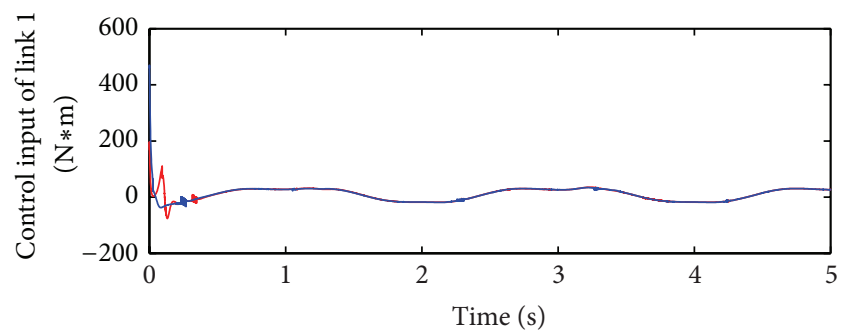

(a)

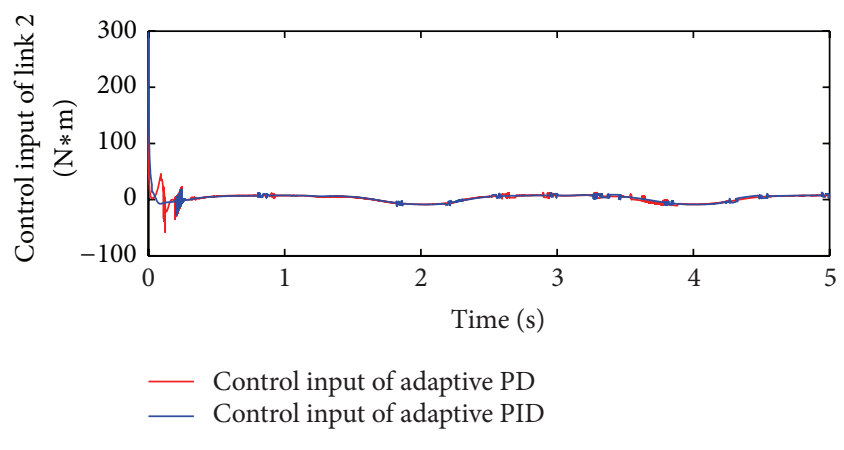

(b)

Figure 10: Control inputs of link 1 and link 2.

provided by the adaptive $\mathrm{PD}$ ones $[25,26]$. Moreover, the robust adaptive PID controllers result in better dynamic performance of the manipulator systems. By observing the experimental figures, it can be verified that the proposed control laws can ensure a faster convergence rate and smaller 


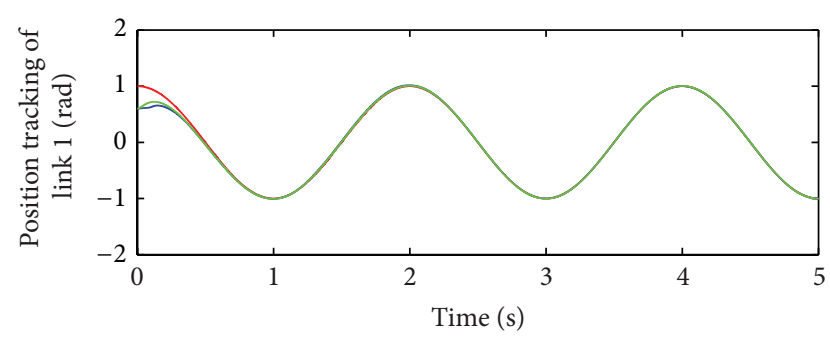

(a)

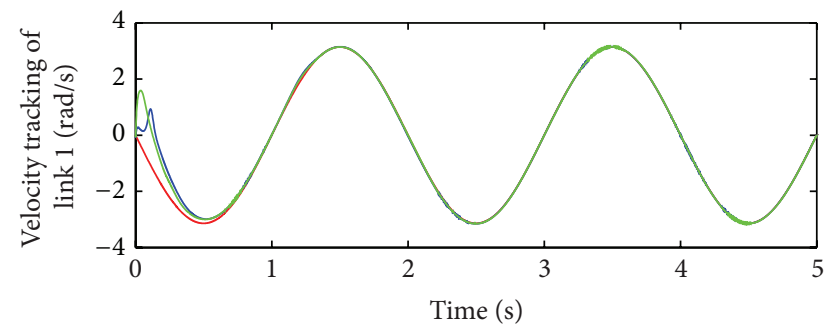

— Desired trajectory
— Actual trajectory of adaptive PD
_ctual trajectory of adaptive PID

(b)

FIGURE 11: Position and velocity trajectory tracking of link 1.

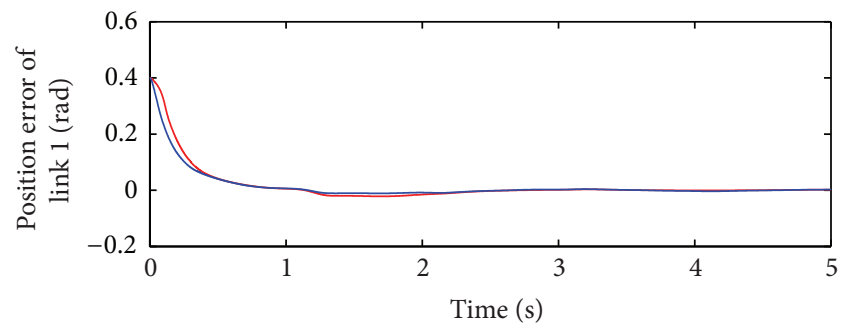

(a)

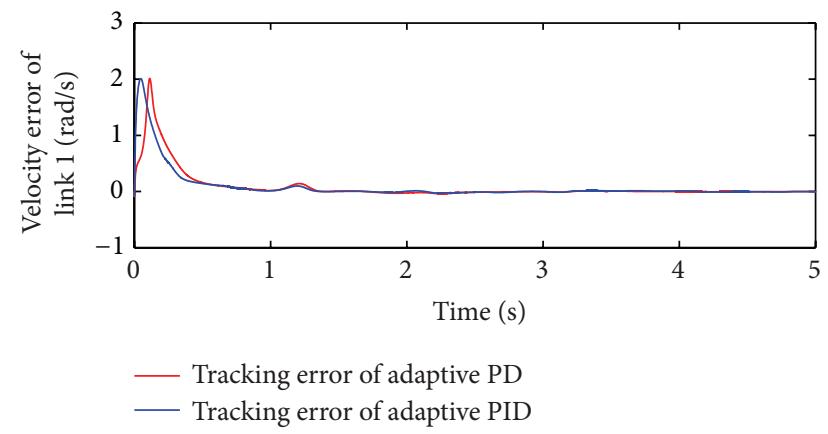

(b)

FIGURE 12: Position and velocity trajectory tracking errors of link 1.

overshoot of the system states tracking to the desired trajectories when compared with the adaptive PD controllers $[25,26]$. In terms of the control input, the robust adaptive PID controllers lead to less chattering effect, which is beneficial to prolong the service life of robot manipulator. For the unknown constant vector $P$, the designed controllers in

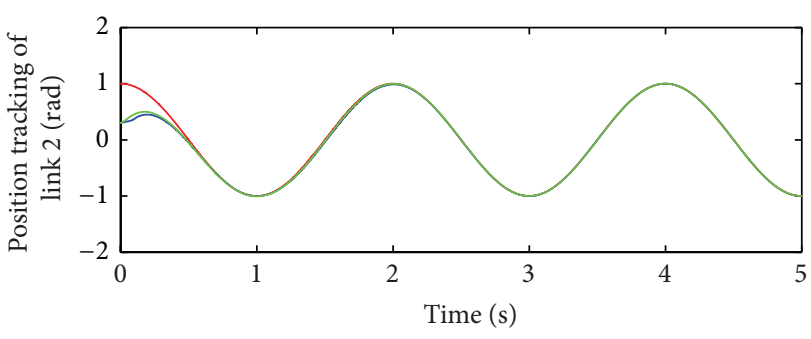

(a)

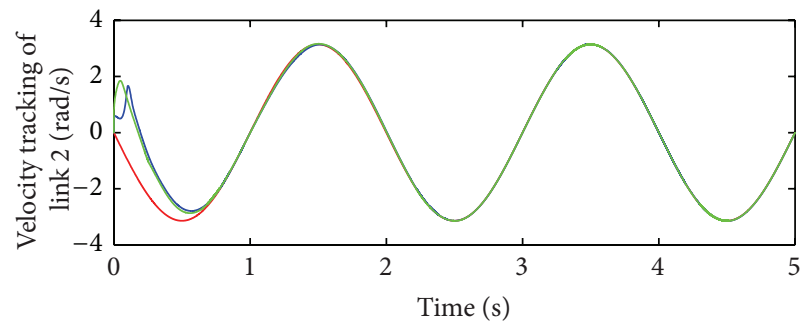

— Desired trajectory
— Actual trajectory of adaptive PD
_ Actual trajectory of adaptive PID

(b)

FIGURE 13: Position and velocity trajectory tracking of link 2.

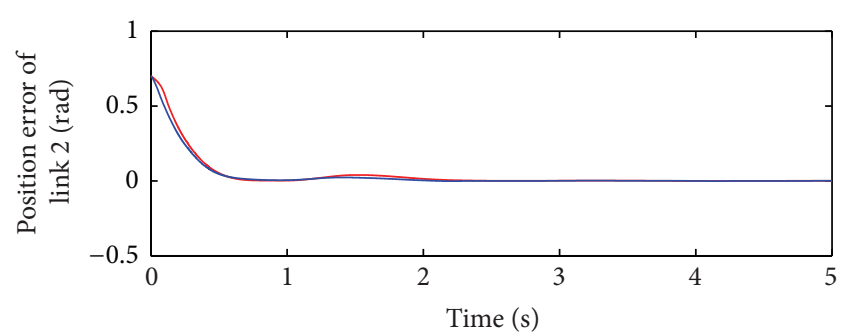

(a)

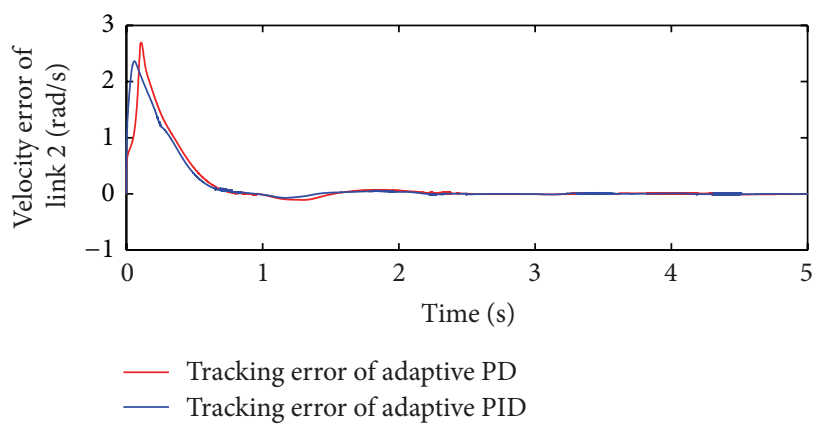

(b)

FIgURE 14: Position and velocity trajectory tracking errors of link 2.

this study ensure higher finite-time estimation accuracy and significantly lower overshoot.

From the above analysis, the investigation of this work presents more effective control methods for robot manipulator operation under unknown bounded external disturbances. 


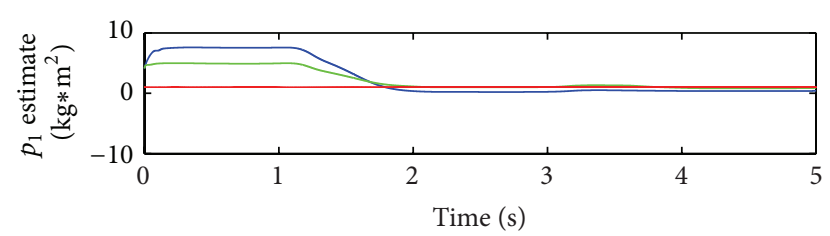

(a)

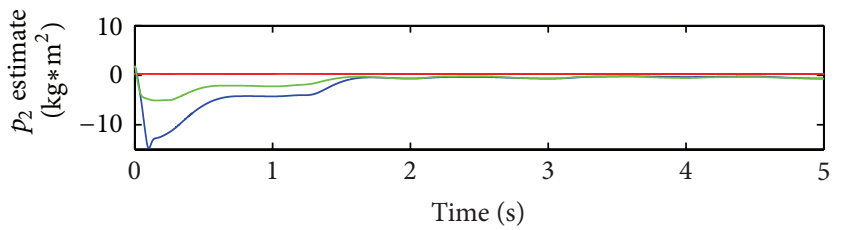

(b)

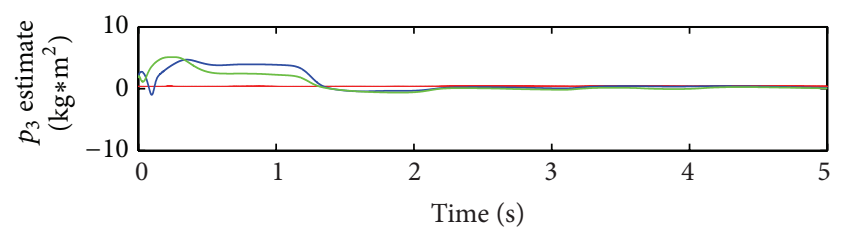

Estimate value of adaptive PD

_ Estimate value of adaptive PID

- Actual value of parameters $p$

(c)

FIgURE 15: Estimate values of parameters $p_{1}, p_{2}$, and $p_{3}$.

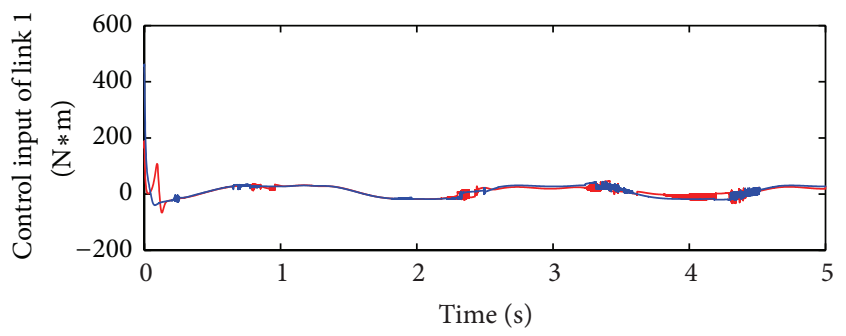

(a)

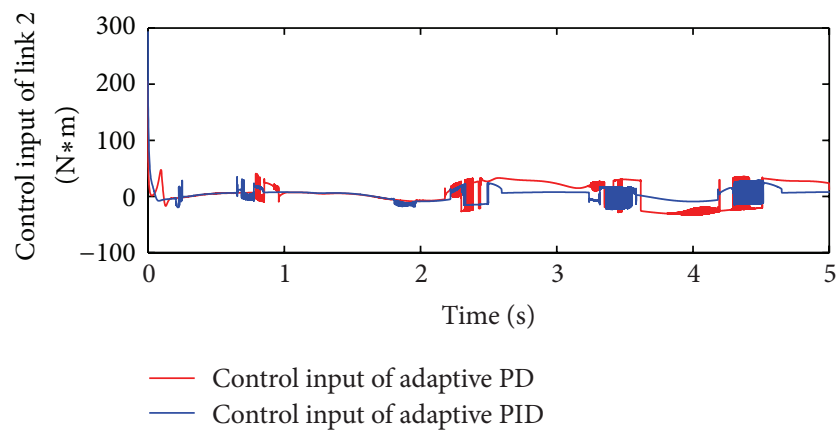

(b)

FIGURE 16: Control inputs of link 1 and link 2.

Remark. Both the adaptive PD control laws in $[25,26]$ and the robust adaptive PID control laws in this study can make the position and the velocity tracking errors of the manipulator systems asymptotically converge to zero, which means $e(t) \rightarrow 0$ and $\dot{e}(t) \rightarrow 0$ as $t \rightarrow \infty$. Therefore, when the control gains chosen for simulation in $[25,26]$ and this paper simultaneously result in rapid convergence of the manipulator positions and velocities, the advantage of the robust adaptive PID controllers will be tiny with comparison to the adaptive PD controllers $[25,26]$.

Nevertheless, it is necessary to emphasize that the proposed controllers are unavailable under manipulator actuators fault. However, actuators fault may occur due to infrequent maintenance or limited life cycle, which could cause several damages to the operators and products. In [31], an adaptive variable structure control scheme is proposed for underactuated mechanical manipulators. Reference [32] investigates the basic data-driven methods for process monitoring and fault diagnosis. And [33] discusses two online schemes for an integrated design of fault-tolerant control systems. Based on these current researches, our future work will focus on the fault diagnosis and fault-tolerant control of robot manipulators in case of actuators fault.

\section{Conclusion}

This paper describes the design and simulation implementation of two new robust adaptive PID controllers for the trajectory tracking control of rigid robot manipulator with known or unknown upper bound of the external disturbances. The main feature of this design is that it combines PID control law with robust adaptive algorithm. The adaptive algorithm is utilized to estimate the unknown constant vector $P$ online, while the robust term and the PID control are used to cope with the unknown bounded external disturbances, adaptive approximation errors, and trajectory tracking errors.

The convergence and stability properties of the closedloop system are guaranteed. In addition, the tolerable external disturbances are enlarged; besides, the dynamic performance of the manipulator systems is improved and the finite-time tracking control accuracy is enhanced by adopting the proposed control schemes in contrast to those achieved through applying the existing adaptive PD control laws [25, 26]. The control techniques have been finally simulated for a planner manipulator model with two rotary degrees of freedom in MATLAB/SIMULINK toolbox. Simulation results show the advantages of the designed controllers and verify that the proposed strategies are able to cope with both the external disturbances, that can typically arise in practical control, and uncertain constant parameters in system dynamics.

The limitation of the proposed control schemes lies in the requirement of $K_{D}=K_{I}$, which in fact constrains the flexibility of the controllers. Currently we are working on the extension of the proposed methods to remove this constraint. Future work will investigate the fault diagnosis and faulttolerant control of robot manipulator systems with actuators fault.

\section{Acknowledgments}

This work was supported by the National Natural Science Foundation of China under Grant 51179038 and the Fundamental Research Funds for the Central Universities of HEUCF041337. 


\section{References}

[1] R. Kelly and R. Carelli, "A class of nonlinear PD-type controllers for robot manipulators," Journal of Robotic Systems, vol. 13, no. 12, pp. 793-802, 1996.

[2] S. Kawamura, F. Miyazaki, and S. Arimoto, "Is a local PD feedback control law effective for trajectory tracking of robot motion?" in Proceedings of the IEEE International Conference on Robotics and Automation, vol. 3, pp. 1335-1340, 1988.

[3] S. Arimoto and F. Miyazaki, "Stability and robustness of PID feedback control for robot manipulators of sensory capability," in Proceedings of the 1st International Symposium on Robotics Research, pp. 783-799, 1984.

[4] E. M. Jafarov, M. N. A. Parlakçi, and Y. Istefanopulos, "A new variable structure PID-controller design for robot manipulators," IEEE Transactions on Control Systems Technology, vol. 13, no. 1, pp. 122-130, 2005.

[5] G. Leitmann, "On the efficacy of nonlinear control in uncertain linear systems," Journal of Dynamic Systems, Measurement and Control, vol. 103, no. 2, pp. 95-102, 1981.

[6] M. J. Corless and G. Leitmann, "Continuous state feedback guaranteeing uniform ultimate boundedness for uncertain dynamic systems," Institute of Electrical and Electronics Engineers, vol. 26, no. 5, pp. 1139-1144, 1981.

[7] M. W. Spong, "On the robust control of robot manipulators," Institute of Electrical and Electronics Engineers, vol. 37, no. 11, pp. 1782-1786, 1992.

[8] E. Yaz, "Commons on "on the robust control of robot manipulators" by M. W. Spong," IEEE Transactions on Automatic Control, vol. 38, no. 3, pp. 511-512, 1993.

[9] K.-M. Koo and J.-H. Kim, "Robust control of robot manipulators with parametric uncertainty," Institute of Electrical and Electronics Engineers, vol. 39, no. 6, pp. 1230-1233, 1994.

[10] M. Danesh, M. Keshmiri, and F. Sheikholeslam, "Developing a robot control scheme robust to uncertain model parameters and unmodeled dynamics," in Proceedings of the 1st IEEE Conference on Industrial Electronics and Applications (ICIEA '06), pp. 1-6, May 2006.

[11] G. Liu and A. A. Goldenberg, "Uncertainty decompositionbased robust control of robot manipulators," IEEE Transactions on Control Systems Technology, vol. 4, no. 4, pp. 384-393, 1996.

[12] J. J. Craig, P. Hsu, and S. S. Sastry, "Adaptive control of mechanical manipulators," International Journal of Robotics Research, vol. 6, no. 2, pp. 16-28, 1987.

[13] J.-J. E. Slotine and W. Li, "On the adaptive control of robot manipulators," International Journal of Robotics Research, vol. 6, no. 3, pp. 49-59, 1987.

[14] R. Burkan, "Design of an adaptive control law using trigonometric functions for robot manipulators," Robotica, vol. 23, no. 1, pp. 93-99, 2005.

[15] R. Burkan, "Modelling of a logarithmic parameter adaptation law for adaptive control of mechanical manipulators," Robotica, vol. 24, no. 4, pp. 523-525, 2006.

[16] K. Y. Lim and M. Eslami, "Robust adaptive controller designs for robot manipulator systems," IEEE Journal of Robotics and Automation, vol. 3, no. 1, pp. 54-66, 1987.

[17] R. Johansson, "Adaptive control of robot manipulator motion," IEEE Transactions on Robotics and Automation, vol. 6, no. 4, pp. 483-490, 1990.

[18] Z. J. Yang, Y. Fukushima, and P. Qin, "Decentralized adaptive robust control of robot manipulators using disturbance observers," IEEE Transactions on Control Systems Technology, vol. 20, no. 5, pp. 1357-1365, 2012.

[19] R. Burkan, "Design parameters and uncertainty bound estimation functions for adaptive-robust control of robot manipulators," Turkish Journal of Electrical Engineering and Computer Sciences, vol. 20, no. 1, pp. 169-186, 2012.

[20] R. Burkan, "Design of adaptive compensators for the control of robot manipulators robust to unknown structured and unstructured parameters," Turkish Journal of Electrical Engineering \& Computer Sciences, vol. 21, no. 2, pp. 452-469, 2013.

[21] F. Aghili, "Adaptive control of manipulators forming closed kinematic chain with inaccurate kinematic model," IEEE/ASME Transactions on Mechatronics, vol. 8, no. 5, pp. 1544-1554, 2013.

[22] D. J. López-Araujo, A. Zavala-Río, V. Santibáñez, and F. Reyes, "Output-feedback adaptive control for the global regulation of robot manipulators with bounded inputs," International Journal of Control, Automation, and Systems, vol. 11, no. 1, pp. 105-115, 2013.

[23] S. I. Han, K. S. Lee, M. G. Park, and J. M. Lee, "Robust adaptive deadzone and friction compensation of robot manipulator using RWCMAC network," Journal of Mechanical Science and Technology, vol. 25, no. 6, pp. 1583-1594, 2011.

[24] M. M. Fateh and S. Khorashadizadeh, "Robust control of electrically driven robots by adaptive fuzzy estimation of uncertainty," Nonlinear Dynamics, vol. 69, no. 3, pp. 1465-1477, 2012.

[25] H. J. Bai, D. F. Tang, and J. B. Cao, "A robust adaptive PD control method of robot manipulator," Journal of Mechanical Transmission, vol. 35, no. 11, pp. 34-40, 2011.

[26] D. Ge and S. Jiang, "Self-adaptive PD control of robot manipulator," in Proceedings of the 8th ACIS International Conference on Software Engineering, Artificial Intelligence, Networking, and Parallel/Distributed Computing (SNPD '07), pp. 605-610, August 2007.

[27] M. W. Spong and M. Vidyasagar, Robot Dynamics and Control, John Wiley, New York, NY, USA, 1989.

[28] J. Slotine and W. Li, Applied Nonlinear Control, Prentice Hall, New Jersey, NJ, USA, 1991.

[29] H. K. Khalil, Nonlinear Systems, Prentice Hall, Upper Saddle River, NJ, USA, 3rd edition, 2003.

[30] C.-Y. Su and T.-P. Leung, "Sliding mode controller with bound estimation for robot manipulators," IEEE Transactions on Robotics and Automation, vol. 9, no. 2, pp. 208-214, 1993.

[31] J. H. Yang and K. S. Yang, "An adaptive variable structure control scheme for underactuated mechanical manipulators," Mathematical Problems in Engineering, vol. 2013, Article ID 270649, 23 pages, 2012.

[32] S. Yin, S. X. Ding, A. Haghani, H. Hao, and P. zhang, "A comparison study of basic data-driven fault diagnosis and process monitoring methods on the benchmark Tennessee Eastman process," Journal of Process Control, vol. 22, no. 9, pp. 1567-1581, 2012.

[33] S. Yin, H. Lou, and S. X. Ding, "Real-time implementation of fault tolerant control system with performance optimization," IEEE Transactions on Industrial Electronics, vol. 61, no. 5, pp. 2402-2411, 2014. 


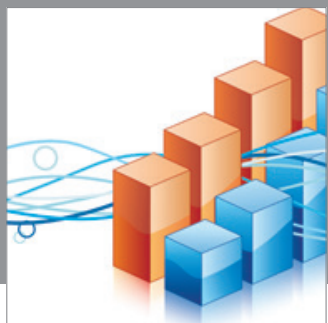

Advances in

Operations Research

mansans

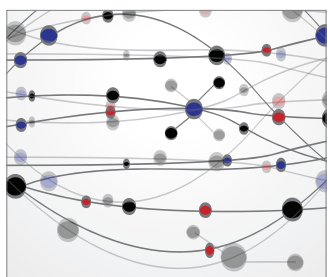

The Scientific World Journal
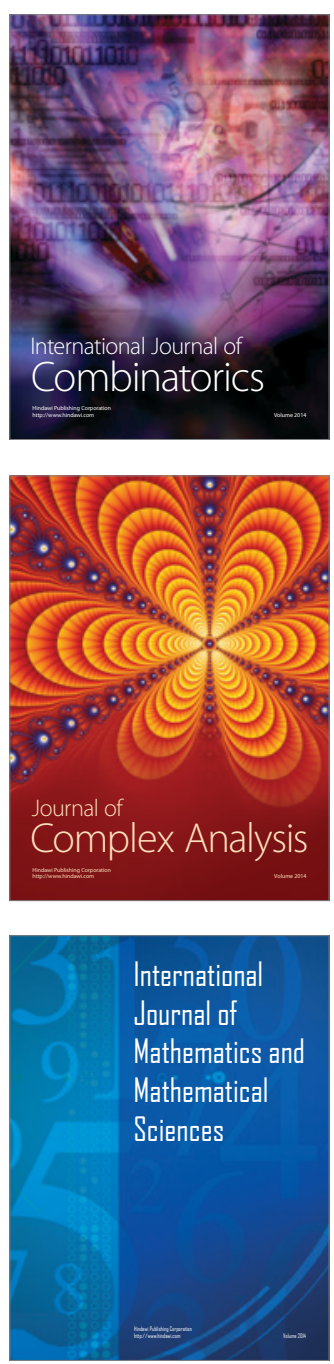
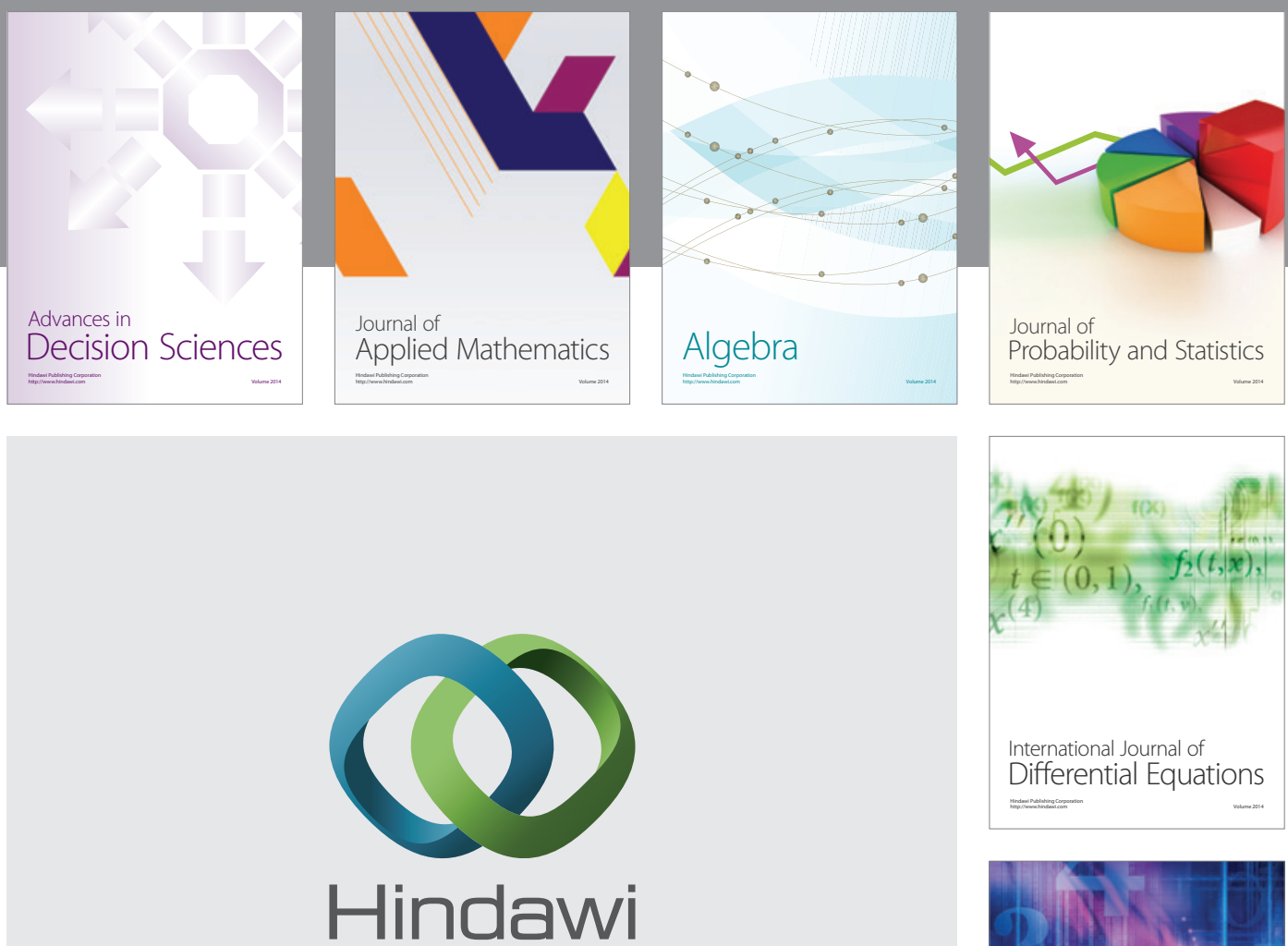

Submit your manuscripts at http://www.hindawi.com
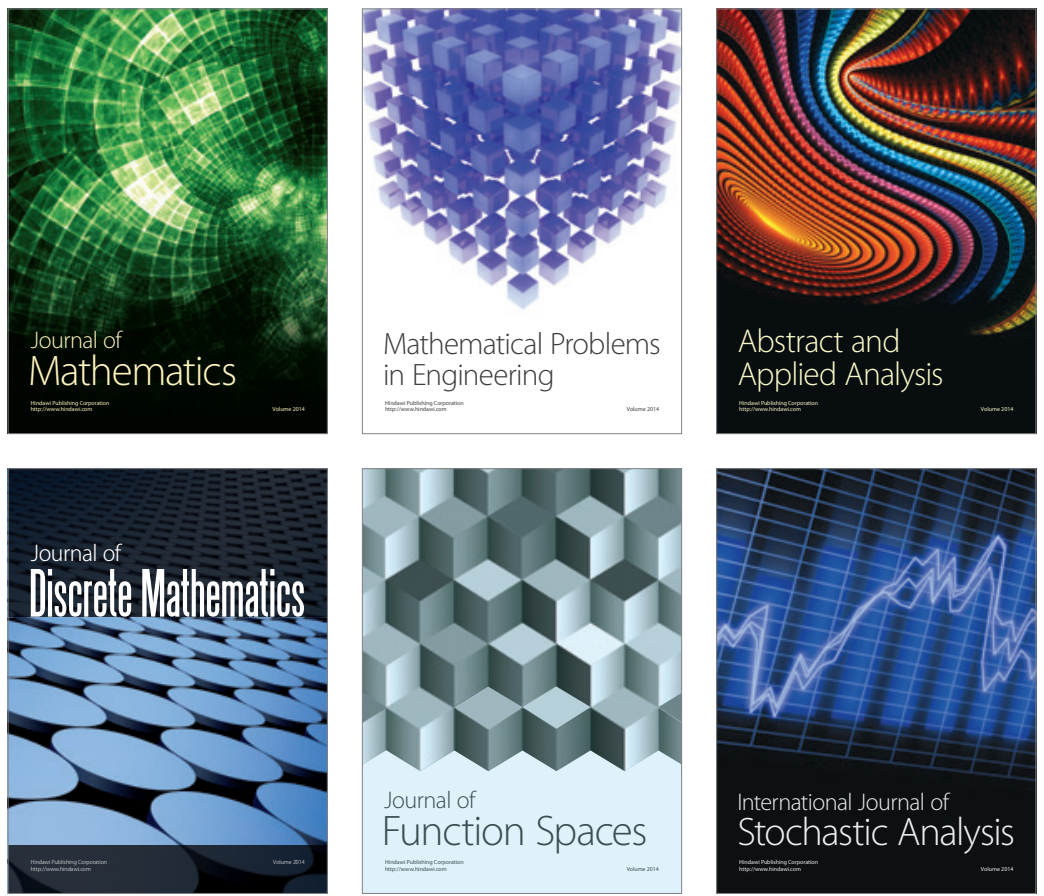

Journal of

Function Spaces

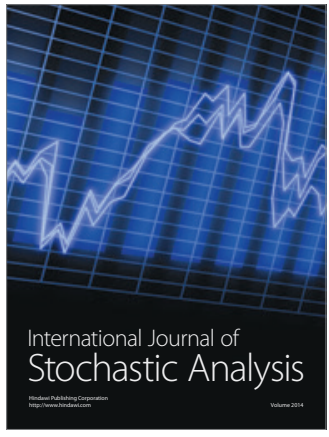

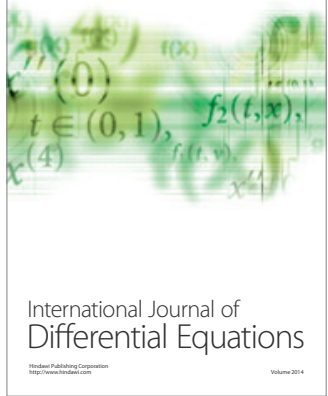
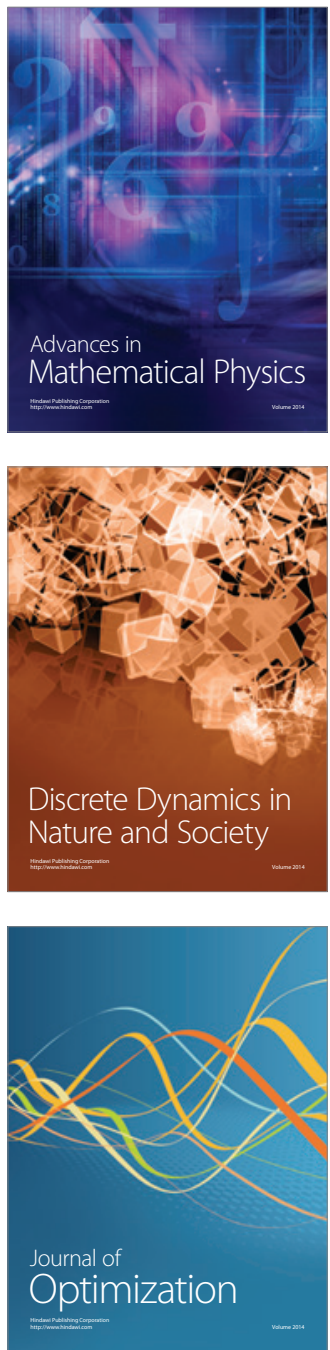